\title{
Éthique de la communication
}

Distinguer ce qu'on a en commun et partager ce que l'on est d'unique pour une communication féconde

\section{Gilles Le Cardinal}

\section{(2) OpenEdition}

12 Journals

Édition électronique

URL : http://journals.openedition.org/communicationorganisation/2676

DOI : 10.4000/communicationorganisation.2676

ISSN : $1775-3546$

Éditeur

Presses universitaires de Bordeaux

Édition imprimée

Date de publication : 1 mai 2002

ISSN : 1168-5549

Référence électronique

Gilles Le Cardinal, « Éthique de la communication », Communication et organisation [En ligne], 21 | 2002, mis en ligne le 19 décembre 2012, consulté le 01 mai 2019. URL : http://journals.openedition.org/ communicationorganisation/2676 ; DOI : 10.4000/communicationorganisation.2676

Ce document a été généré automatiquement le 1 mai 2019.

(c) Presses universitaires de Bordeaux 


\title{
Éthique de la communication
}

\author{
Distinguer ce qu'on a en commun et partager ce que l'on est d'unique \\ pour une communication féconde
}

\section{Gilles Le Cardinal}

\section{Introduction}

1 La démarche que nous proposons pour une réflexion sur l'éthique de la communication interpersonnelle part du texte de la Genèse en passant par la psychanalyse et l'anthropologie pour aboutir aux sciences de l'information et de la communication. Ces dernières sont transdisciplinaires par principe, quand elles prennent en compte les dimensions de la relation interpersonnelle et des relations sociales.

2 C'est dans la Genèse que nous irons chercher les racines de la question éthique conçue comme science de la connaissance du bien et du mal et nous tenterons de l'appliquer à la communication. Notre question peut se formuler ainsi: Qu'est-ce que communiquer «bien » et qu'est-ce que communiquer « mal »?

3 Pour répondre à cette interrogation, nous proposons une approche anthropologique décrivant les différentes dimensions de l'être humain. L'éthique, science du discernement, doit à la fois clarifier des valeurs et déboucher avec cohérence sur une mise en pratique de préconisations applicables et de principes généraux sans toutefois tomber dans le normatif étriqué. Mais avant de définir le «bien» ou le «mal» communiquer, il nous faudra d'abord donner des définitions claires de la communication interpersonnelle, de l'autonomie, de l'interaction.

4 Nous nous appuierons enfin sur une modélisation de la communication en quatre processus principaux pour déduire des pièges repérés, des principes éthiques et des conseils pratiques. 


\section{Fondement et problématique de l'éthique de la communication}

5 La communication commence par l'acte de donner un nom. préalable indispensable pour appeler quelqu'un par son nom.

Ce premier acte de parole est un acte de différenciation. Un nom permet d'identifier un être, de ne pas le confondre avec un autre, de le reconnaitre parmi d'autres. Un nom est la réalité symbolique qui nous permet de faire mémoire d'une personne, même quand elle n'est pas là.

7 Une différence entre deux noms, reflet d'une différence entre deux êtres va permettre de se comporter différemment avec l'un et avec l'autre. Cette différence informe d'une spécificité, d'une identité unique. Cette différence est information. Une information, dit Gregory Bateson ${ }^{1}$, est une différence qui fait une différence.

Juste après la création de l'homme, («ish», en hébreu), va s'opérer un acte de différenciation nous dit la Genèse. "Isha", la femme, l'autre côté de l'être humain, comme le dit Annick de Souzenelle², sera séparée de «Ish». Elle permettra à Adam de sortir, à sa demande, de la solitude car « Il n'est pas bon pour l'homme d'être seul! »

Yahvé dit :

" De tout arbre du jardin, tu mangeras »,

grande liberté qui est assurée grâce à cette exception, ce « partout sauf » qui la fonde :

« mais de l'arbre de la connaissance du bien et du mal, tu ne mangeras pas ».

11 Est-ce la connaissance du bien et du mal, fondement de l'éthique que le Dieu de la Bible il serait alors pervers - interdirait ainsi à l'homme? La construction de la phrase est pourtant assez claire, semble-t-il, pour dire que cette interprétation est inexacte. C'est l'acte de manger qui est interdit, l'acte de manger l'arbre de la différenciation du bien et du mal. Manger, objet de l'interdit, c'est l'acte indifférentiateur, par excellence. L'acte qui annihile une différence pour confondre, pour fondre ensemble le moi et le non-moi, la nourriture.

12 L'interdit fondamental n'est donc pas, à mon sens, la connaissance du bien et du mal et je suis en cela Marie Balmary, mais bien de manger la différence, de confondre en moi le bien et le mal, le «connaître bien » et le «connaître mal », ou vouloir le décréter sans tenir compte des lois universelles.

13 La Bible, loin d'empêcher la réflexion sur l'éthique, est dans un sens, ce qui a permis de la fonder par l'énoncé d'un seul interdit, celui de «manger les différences ». de confondre le bien et le mal.

Marie Balmary ${ }^{3}$ synthétisera cette réflexion dans une phrase fulgurante : «Il n'y a qu'un interdit, manger l'autre».

René Girard ${ }^{4}$ parlera lui, de désir mimétique qui caractérise l'être humain, mais qui peut aussi être la source de nos difficultés relationnelles et qui peut engendrer les violences les plus inhumaines. Le désir mimétique, c'est le désir pour soi d'être comme l'autre; il commence par le désir de l'objet que l'autre possède, de la place ou du rôle qu'il tient, de l'amour qui l'anime, pour culminer dans le désir du désir de l'autre. 
16 Vouloir « l'autre comme soi » ou n'accepter que « les autres comme soi », est à la source de tous les racismes ; «Vouloir être comme l'autre » est à la source de toutes les névroses, confusion et chaos de l'âme.

17 Le désir de l'autre survient du plus profond du cœur de l'homme. Une fois l'autre apparu, c'est sur ce désir mimétique que va jouer le serpent, figure de Satan, l'accusateur, pour tenter Ève (la première différenciée, dit Marie Balmary) à transgresser l'interdit de la confusion du bien et du mal, en disant, « Vous serez comme... des dieux ». (Gen.3, 5)

18 Voyons comment, dans la Genèse, les sentiments se succèdent rapidement : appel à la vie par un autre, désir de l'autre immédiatement suivi de la tentation "d'être comme », de « manger l'autre ». Puis juste après la transgression de l'interdit naît la peur.

19 À Yahvé qui lui demande :

«Où es-tu?»

20

Adam répond :

«J'ai entendu ton pas dans le jardin ; j'ai eu peur parce que je suis nu et je me suis caché ». (Gcn. 3, 9, 10)

21 - Désir d'un autre (« il n'est pas bon que l'homme soit seul »)

22 - Tentation d'être comme... (« vous serez comme des dieux »)

23 - Peur de la relation, qui naît juste après la transgression de l'interdit, de manger la différence entre soi et l'autre.

24 Comment vivre ensemble dès lors que ces trois sentiments cohabiteront à jamais dans l'homme, désir, tentation, peur? Comment stabiliser la coopération interpersonnelle, l'harmonie sociale, la paix entre les sociétés, les cultures? Telle est la question posée par l'éthique de la communication.

\section{Approche anthropologique de l'être humain}

\section{L'être humain unique et défaillant}

\section{Corps}

25 Notre corps est unique, cela est inscrit dans chacune des milliards de cellules qui constituent ce corps dans le patrimoine génétique. Cela se traduit par un visage, un regard, des empreintes digitales uniques. Mais ce corps unique est aussi défaillant ce qui implique, dans une perspective éthique :

26 - Le respect du corps, de sa place, de sa sensibilité, de ses souffrances,

27 - L'attention portée à ses blessures, ses maladies, ses handicaps, ses défaillances, sa finitude, sa mort,

28 - La prise en compte de sa santé, de son plaisir, de ses performances, de son désir de fécondité.

29 - La valeur qu'incarne le corps est « la vie» 


\section{Intelligence}

30 Notre intelligence est unique, inscrite dans un cerveau dont la structure générale est la même chez tous les êtres humains, mais le détail du câblage neuronal du cerveau dépend de chacune de nos expériences, de chacun de nos apprentissages.

31 Le caractère unique et défaillant de l'intelligence humaine implique :

32 - Le respect de ses connaissances, compétences, capacités d'apprentissage, de ses émotions, de ses valeurs, de sa culture, de son expérience,

33 - L'attention portée à ses limites cognitives, ses difficultés d'apprentissage, ses blocages, ses échecs, ses déviances, ses aveuglements, ses ignorances,

34 - La prise en compte de ses motivations, de ses projets, de ses réussites, de ses découvertes.

35 - La valeur que sert l'intelligence est « la vérité».

\section{Relation}

36 Notre vie relationnelle est unique, comme notre réseau relationnel tel qu'il s'inscrit dans notre agenda. Notre façon d'entrer en relation, d'attribuer notre confiance, d'aimer est unique. Mais notre vie relationnelle unique est aussi défaillante, ce qui implique :

37 - Le respect des proches d'une personne, de ses amitiés, de ses réseaux, de ses engagements, de ses alliances,

38 - L'attention portée à ses difficultés relationnelles, conflits, trahisons, représailles,

39 - La prise en compte des risques pris ensemble, des promesses faites, des coopérations réussies, des pardons échangés, de la confiance que nous faisons à l'autre et celle qu'il nous fait.

$40-$ - Amour et confiance» sont les valeurs qui caractérisent la relation interpersonnelle.

\section{Identité}

41 Notre identité, notre personnalité est unique, notre façon de porter en nous les êtres absents (notre vie spirituelle), nos valeurs, nos croyances, nos appartenances sont uniques. Mais notre identité unique est aussi défaillante, ce qui implique :

42 - Le respect de l'histoire d'une personne, de sa filiation, de sa parenté, de ses valeurs, de ses croyances, de ses appartenances sociales et de son rôle social,

43 - L'attention portée aux confusions possibles, à ses désirs mimétiques, à sa jalousie, à ses manques de confiance en lui, à ses tentations d'orgueil, de pouvoir sur les autres,

44 - La prise en compte de ses spécificités et talents, de son œuvre, de sa famille, de sa descendance, des qualités propres à son être, en un mot de son unique

45 - «L'estime de soi et la reconnaissance des autres » sont les valeurs associées à la dimension identitaire de la personne.

\section{L'être humain libre et autonome}

A ces valeurs doivent être associées la liberté et l'autonomie. Être libre, c'est avoir des marges de manœuvre dans un champ de contraintes et assumer ses choix. 


\section{4 - ne pas choisir à la place de l'autre}

55 L'attention aux contraintes qui pèsent sur l'autre, à la continuité et la cohérence de ses choix, à ses échecs, à ses aliénations, à ses dépendances et addictions :

56 - Identifier les aliénations, les dépendances (de soi et des autres) pour initier un travail de libération

La prise en compte et la diversification des choix possibles, l'accroissement des marges de manœuvre, la cohérence et la bonne définition de ses objectifs, l'accès aux moyens de passer à l'acte efficacement, le respect de ses engagements, le développement de ses capacités d'apprentissage :

\section{L'être humain interactif et communiquant}

Pour bien comprendre les enjeux de l'interaction de deux êtres différents et libres, il est crucial de disposer d'une bonne définition qui caractériserait la structure unité d'interaction. Pour cela, mettons en présence deux acteurs libres, c'est-à-dire possédant au moins deux choix possibles et respectant la liberté de l'autre, c'est-à-dire en ne choisissant pas à sa place. Il en résulte qu'ils vivront l'un des quatre événements possibles sans pouvoir se désolidariser une fois leur choix posé, comme l'indique le schéma « unité d'interdépendance ».

\begin{tabular}{|l|c|c|}
\hline \multicolumn{1}{|c|}{ Tu } & Choix 1 & Choix 2 \\
\hline Choix 1 & $\mathrm{e}_{1}$ & $\mathrm{e}_{2}$ \\
\hline Choix 2 & $\mathrm{e}_{3}$ & $\mathrm{e}_{4}$ \\
\hline
\end{tabular}


Unité d'interdépendance d'interaction comme: les stratégies dominante, prudente, d'imitation (faire comme l'autre à la séquence précédente, stratégie donnant-donnant ${ }^{5}$ ), pavlovienne (décrite par Nowak et Sigmund ${ }^{6}$ " je gagne, je continue - je perds, je change »), opportuniste (saisie de toutes les occasions même au détriment de l'autre) ou la stratégie fondée sur la gestion de la confiance : principe de plus ou moins grande prise en compte du revenue de l'autre par un couplage de revenus de plus en plus fort (« on gagne ensemble ou on perd ensemble ${ }^{7}$ »). «Tenir compte de l'autre » est un grand invariant que l'on retrouve dans toutes les cultures pour réguler les interactions.

\section{L'éthique de l'interaction}

En situation d'interaction, aucun des deux acteurs ne peut atteindre par sa volonté, un événement préféré car il dépend du choix fait par l'autre. Il nous parait essentiel pour construire une éthique de l'interaction, de bien identifier cette structure d'interaction des libertés conduisant à un événement où les acteurs sont solidaires mais ne subissent pas les mêmes conséquences, et n'ont pas les mêmes préférences.

\section{l'énoncé}

71

otre premier conseil consiste donc à modéliser la structure d'interaction pour mieux la comprendre et anticiper les difficultés qu'elle engendre.

On déduira alors les pièges contenus dans cette structure d'interaction comme :

L'accusation mutuelle d'être à l'origine d'une situation indésirable pour tous les acteurs.

Le paradoxe des stratégies dominantes et prudentes, apparemment souhaitables mais qui conduisent finalement à ne pas coopérer,

L'instabilité de la coopération et la stabilité de la non-coopération mutuelle qui sont démontrées dans le cas du dilemme du prisonnier

Le deuxième conseil consiste à identifier les risques de trahison contenus dans toute situation pour pouvoir les gérer de façon adéquate.

Pour cela, nous proposons d'identifier les dilemmes présents et souvent cachés par Le troisième conseil propose de se mettre en pensée à la place de l'autre pour ne pas prendre sa place dans les faits. 
72 Le problème majeur de l'interaction consiste à stabiliser la coopération et à éviter la trahison surprise et la non-coopération mutuelle si fréquente. Plusieurs stratégies sont alors possibles : Les représailles associées à toute trahison ou toute transgression: c'est le rôle de la justice fondée sur le droit; Le couplage des revenus comme le suggère la stratégie de la confiance qui implique le courage d'avoir peur de la trahison, qui reste toujours possible ; Le quatrième conseil nous engage à bien gérer les confiances. Ce qui peut être considéré comme une nouvelle vertu ${ }^{9}$ entre le courage d'avoir peur et la justice qui dénonce les transgressions. Elle propose de se coupler à l'autre jusqu'à un certain niveau de risque.

\section{Information et communication}

Nous proposons une première définition de la communication sous la forme suivante :

Communiquer c'est échanger de l'information pour pouvoir vivre et agir de façon adaptée à un monde de manière à réaliser des projets en relation avec les autres.

\section{Qu'est-ce que l'information?}

7 L'information est ce qui nous permet de sortir de l'incertitude, de percevoir une différence cachée.

Henri Laborit ${ }^{10}$, dans son livre "La nouvelle grille», distingue utilement l'information circulante de l'information structure.

L'information circulante est un ensemble de signaux sonores, lumineux, tactile, olfactif, électronique, qui circulent entre l'émetteur et le récepteur. Ils sont alors objectifs, reproductibles, mesurables, quantifiables. Objet pour les sciences physiques par conséquent, mais objet dépourvu de sens.

L'information structure au contraire, est le système engrammé dans le cerveau qui permet de décoder et d'interpréter une information circulante et de générer ainsi une signification. L'information structure est unique, subjective, partiellement inconsciente. Elle demeure à jamais hors du champ scientifique car unique, non reproductible.

81 Mais heureusement, les cerveaux de tous les êtres humains ont cependant des caractéristiques communes: réseaux neuronaux, zones consacrées à la parole, la motricité, les émotions, la logique. Nous sommes tous soumis à la pesanteur, aux besoins de nourriture, de chaleur, de protection, nous avons un langage, une culture propre à notre groupe humain, c'est ce que Jurgen Habermas ${ }^{11}$ appelle «le monde vécu ». Les informations structures, formées par une combinaison de la génétique d'une part, et de nos expériences de vie mémorisées d'autre part, ont une partie commune que j'appelle «le fonds commun d'évidences».

Dès que deux personnes se rencontrent, un fonds commun d'évidences les relie. Elles vont peu à peu le découvrir, pour le transformer alors en "Common Knowledge ${ }^{12}$ ", connaissances partagées par deux ou plusieurs acteurs. Un des objets de la rencontre sera d'identifier et d'augmenter le fonds commun d'évidences, partie commune des informations structures. 


\section{Qu'est-ce que communiquer?}

Une première fonction de la communication interpersonnelle consiste à découvrir et à construire le fonds commun d'évidences.

C'est le socle de la communication toujours à consolider et à faire grandir, autour duquel se révèlent les différences.

Or, dès que nous pensons "différence ", nous aimerions y accoler le mot richesse, mais nous voulons oublier qu'elle est aussi souffrance. La différence ressemble à Janus, ce Dieu à double face, car elle est à la fois richesse et souffrance.

Le psychanalyste Denis Vasse ${ }^{13}$ insiste sur le côté souffrant de la différence en nous faisant comprendre que la souffrance de la différence n'est acceptable que dans la joie de la reconnaissance. Nous ajouterons que cette joie n'est durable que s'il y a fécondité de la coopération de ces différences. Or cela est très difficile! D'où la tentation de manger cette différence qui nous fait tant souffrir, plutôt que de reconnaitre cette différence car cela implique d'accepter la souffrance qui l'accompagne. Nos échanges d'information ne cessent de nous dire que nous sommes différents, tout simplement parce que chacun de nous est unique.

La rencontre de deux êtres uniques génère par définition de la différence, de l'information, donc de la souffrance et de la joie. Communiquer, consiste après avoir identifié le fonds commun d'évidences entre nous, à découvrir nos différences irréductibles en nous aidant à reconnaître nos uniques, nos spécificités, nos singularités. Sans interaction, pas de possibilité de découvrir notre identité unique, ni celle de l'autre.

La communication est donc l'occasion de dépasser la souffrance de la différence pour accéder à la joie de mieux se connaître, de mieux reconnaître nos différences, de mieux identifier nos identités uniques. Le choc de la rencontre nous fait toucher du doigt ce que je suis et que l'autre n'est pas, ce que l'autre est et que je ne suis pas.

Or, nous sommes dotés de cette capacité à accepter un autre différent de nous, à lui attribuer des connaissances, des intentions, des désirs qui ne sont pas les nôtres, à nous y intéresser, au-delà de la remise en cause de nous-mêmes, parfois douloureuse, qui s'opère ainsi.

\section{Se mettre à la place de l'autre pour ne pas prendre sa place}

Erol Franko ${ }^{14}$, dans une intervention au colloque « Du désastre au désir », en s'appuyant sur la théorie de l'esprit, définit la « cécité mentale » comme l'incapacité pour quelqu'un d'attribuer à l'autre des désirs différents des siens, l'incapacité à imaginer les intentions qui guident les actions.

1 Il raconte l'expérience dite de « la boîte de Smarties ».

92 Sophie et Bérénice regardent Jean sortir une boîte de Smarties de sa poche. Il leur demande : «que contient d'après vous, cette boîte ?» «Des Smarties !» répondent-elles ensemble.

Jean ouvre la boîte, vide les Smarties sur la table puis les réintroduit dans la boîte qu'il referme. Il fait sortir Sophie. Devant Bérénice, il vide la boîte dans sa poche, y introduit un crayon et la referme. Il fait revenir Sophie et demande à Bérénice : «Si je demande à Sophie ce qu'il y a dans la boîte, que me répondra-t-elle?» 
Or, nous apprend Paul Watzlawick ${ }^{15}$ "tout événement ou toute situation peut être recadré positivement ou négativement ». Suis-je capable, si je recadre un événement positivement, de comprendre que l'autre puisse en construire, lui, une représentation négative.

Prenons l'exemple de l'image de la figure 1 à double signification.

On peut toujours y voir une vieille femme ou une jeune femme. Il faut confronter deux interprétations différentes pour rentrer véritablement dans l'intelligence de cette image, pour en découvrir la complexité à travers sa double signification.

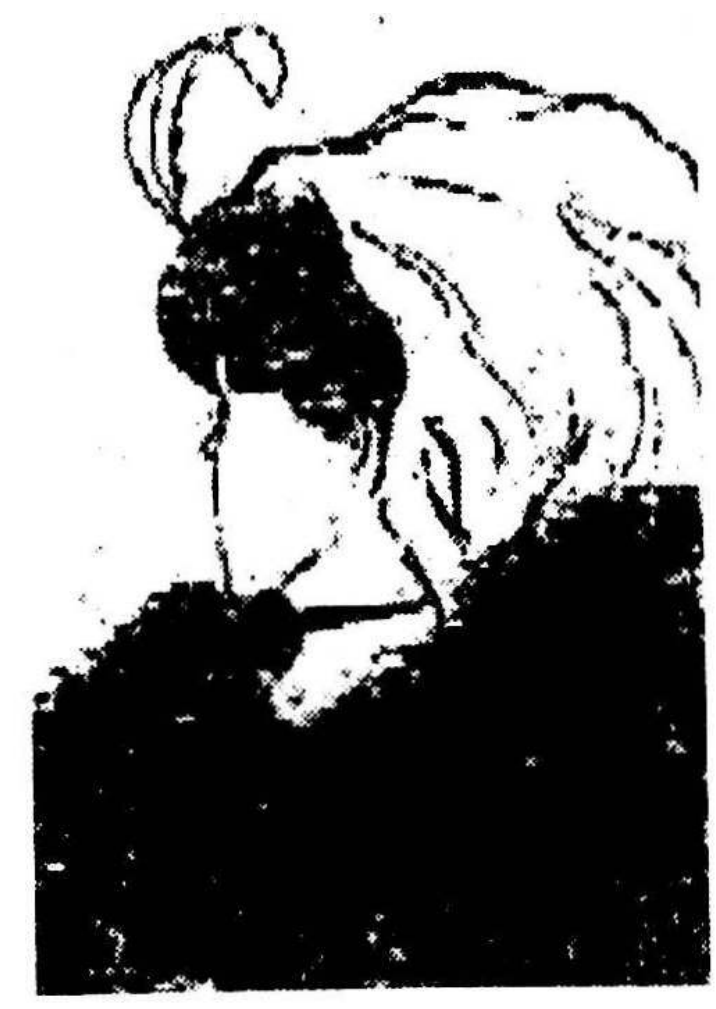




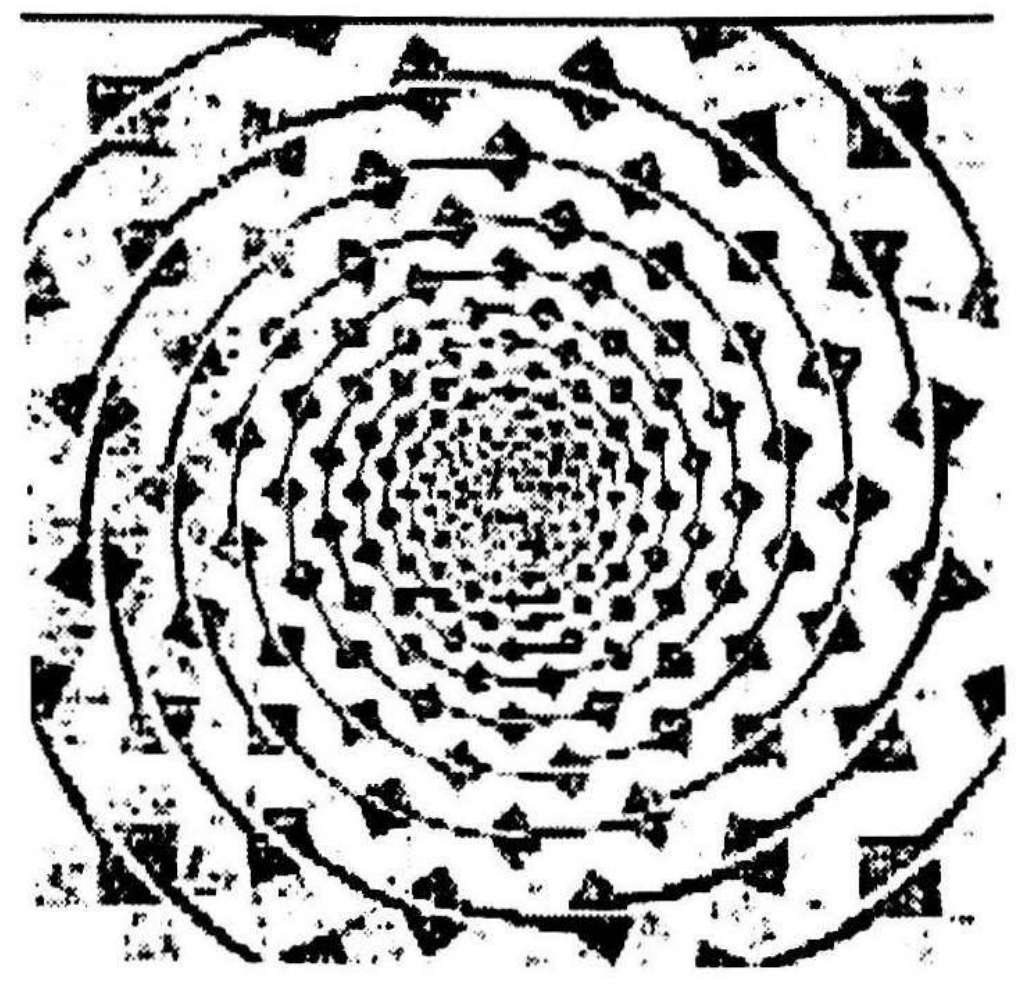

Fig 2 : La spirale de Frazer

99 La spirale de Frazer (fig 2) prend une interprétation différente suivant qu'on l'observe ou que l'on cherche à agir pour suivre la figure avec un crayon. Spirale pour l'observateur, cercles concentriques pour le dessinateur. Nous découvrons ainsi que la représentation construite par un observateur diffère de celle construite par un acteur. Différences de regards, différences de postures entraînent des différences de représentation, des différences dans l'action.

\section{Définition de la communication}

100 À la lumière de ces considérations, nous pouvons maintenant proposer une nouvelle définition de la communication interpersonnelle :

«Communiquer c'est à la fois identifier et construire un fonds commun d'évidences, découvrir des différences - sans les annuler pour éviter de souffrir -, utiliser ces informations pour mieux identifier nos uniques, nos identités grâce à cet autre regard que la présence de l'autre aide à porter sur les événements et les personnes».

«Communiquer c'est enfin gérer l'interaction de personnes uniques dont la coopération peut engendrer la nouveauté, l'innovation, l'invention ».

101 Si les différences se combinent, s'unissent, interagissent positivement, elles peuvent donner du fruit à l'image du masculin et du féminin, qui, par leur union, peuvent procréer un être nouveau, unique.

Or, d'après Erol Franko : «toute personne se comporte de la façon la plus satisfaisante compte tenu de sa représentation du monde». Comprendre le comportement de 
quelqu'un implique donc de connaître ses représentations du monde, ses espérances de gain, ses peurs, et ses tentations pour espérer connaitre ses préférences et anticiper ses choix.

Faire évoluer une personne, en respectant sa liberté, implique donc de lui donner les moyens de d'abord changer ses représentations. Cela peut se faire en lui donnant de nouvelles informations, en lui faisant découvrir de nouvelles valeurs et d'autres ressentis que les siens.

\section{Éthique de la communication interpersonnelle}

104 Pour terminer cette réflexion, nous voudrions descendre au niveau le plus pratique. Pour cela, nous allons passer en revue les fonctions de la communication identifiées dans notre travail de modélisation de la communication intitulé «l'homme communique comme unique $^{16}$ » pour essayer d'en extraire les principaux pièges, quelques principes et proposer quelques précautions utiles à une communication de qualité. L'éthique pratique ne consiste-t-elle pas à utiliser une communication de qualité au service de bonnes finalités?

\section{Le transfert d'information}

\section{Problème technique du transfert d'information}

Pour une bonne qualité du transfert d'information, il est important de veiller au choix du codage, à la puissance et au rythme d'émission, au niveau du bruit dans le canal de transmission, en tenant compte de la capacité de décodage et de réception du destinataire et de sa place.

Les pièges sont constitués par la mauvaise connaissance du récepteur (et ses handicaps éventuels), la multimodalité de l'émission, notamment son caractère digital (la langue) et analogique (le comportement), la présence permanente possible de double message (antagonisme du message digital et du message analogique).

107 Le principe que nous dégageons pour cette fonction de la communication consiste à bien adapter l'émission à la réception, et à rendre cohérent le dire et le faire.

Une précaution simple permet de minimiser certains risques d'erreurs : faire répéter le message transmis par le destinataire.

\section{Problème sémantique}

La communication est complexe par le fait qu'il y a toujours deux constructions de sens : celle de l'émetteur et celle du récepteur. Cela nous conduit à devoir bien identifier l'information structure du destinataire et le fonds commun d'évidences qui existe entre lui et l'émetteur.

110 Pour éviter les pièges de l'ambiguïté sémantique, nous proposons le principe suivant:

111 - Essayer d'accéder aussi bien à l'interprétation de l'émetteur qu à celle du récepteur en se mettant à la place de l'autre, tout en sachant que cet effort est toujours imparfait et sans cesse à reprendre, ce qui permet parfois de construire un sens nouveau. 
112 La précaution qui permet de vérifier la qualité du travail ainsi réalisé est de faire reformuler les énoncés importants par le destinataire, ce qui permet de découvrir certaines distorsions d'interprétations.

\section{Pilotage de l'action}

113 Piloter l'action se heurte toujours au dilemme de la coopération. Chacun a en permanence le choix de coopérer ou de ne pas coopérer. On peut l'éviter en décidant de faire seul, mais c'est rarement possible. Faire ensemble pose un certain nombre de problèmes: relation d'égalité ou de supériorité, dilemme contribution-rétribution, risque de mauvaise coordination, écart dans l'évaluation.

114 Le piège du non-respect de la liberté de l'autre est permanent. La méconnaissance de la structure d'interaction, forme particulière de cécité mentale, est omniprésente. On est tenté de faire à la place de l'autre, de vouloir imposer à l'autre un changement. Trop souvent on ignore la fragilité de la coopération et les précautions nécessaires à sa stabilisation.

115 Le principe que nous pouvons proposer, pour mieux communiquer dans l'action peut s'énoncer ainsi :

116 - Si l'on veut faire évoluer l'autre et évoluer soi-même, la meilleure stratégie est de lui donner les moyens de complexifier nos représentations du monde. Pour cela il faut échanger autant que l'on peut à propos des informations, des émotions, des valeurs et des représentations que possèdent les différents acteurs de manière à construire, à partir de là, une représentation commune. La précaution à prendre ici est de ne laisser passer aucune trahison sans la mettre en mots de manière à la mettre en lumière. Il est bon de ne pas oublier que la construction de la confiance se fait souvent à partir des erreurs à condition qu'elles soient reconnues et retravaillées ensemble.

117 La coopération n'existe que fondée sur une représentation construite en mettant en commun informations, valeurs et émotions, de manière à faciliter la réalisation d'un projet défini ensemble.

\section{La création de relation}

118 Comme le dit Jurgen Habermas ${ }^{17}$, nous n'agissons pas en effet uniquement en fonction de buts prédéterminés mais bien grâce aussi à une compréhension du monde médiatisée par la langage. Paul Watzlawick ${ }^{18}$ met en évidence que la communication joue sur deux tableaux, l'échange d'information et la création de relation.

119 Les relations interpersonnelles ne sont pas seulement au service de l'action, mais visent à l'intercompréhension.

120 La qualité de la relation se traduit par un niveau de confiance qui peut conduire à deux stratégies erronées: une trop grande naïveté, une trop grande méfiance qui l'une et l'autre encouragent la non-coopération.

121 Les pièges de la relation sont bien connus : maximisation du profit à court terme qui pousse à la trahison, la méfiance réciproque qui stabilise le conflit. Le principe suivant propose de porter notre attention sur les processus de construction et de déconstruction de la confiance interpersonnelle. 
Cela suppose le respect de la liberté de l'autre donc de le délier de notre pouvoir sur lui pour espérer avoir avec lui des relations coopératives en acceptant de prendre des risques proportionnés à la confiance qui nous lie ensemble.

Les bonnes précautions à prendre consistent à identifier à l'avance si possible, les paradoxes de la relation et les double binds pour prévenir ou dénoncer leur apparition, et là encore, à retravailler ensemble les défaillances constatées lorsqu'on n'a pas pu les éviter.

\section{Découvrir et construire son identité}

Dans tout message se cache le dialogue suivant, nous dit Paul Watzlawick ${ }^{19}:$ «Voilà comment je me vois, voilà comment je te vois, voilà comme je crois que tu me vois. »

Or, on peut toujours construire de l'autre une image positive (l'unique) ou négative (le défaillant). La souffrance de la différence d'identité peut nous conduire à l'exclusion ou au mimétisme. L'indifférenciation conduit à la violence.

Un bon principe consiste alors à accepter puis à dépasser la souffrance de la différence inhérente à toute rencontre entre deux uniques, pour espérer atteindre la joie de la reconnaissance mutuelle. Ainsi parviendrons-nous peut-être à nous révéler les uns les autres. Cela passe par la mise de notre don au service de la défaillance de l'autre pour espérer voir l'autre en retour mettre son talent au service de notre défaillance. En cas de conflit, il est conseillé de recourir à un tiers médiateur qui permet de restaurer un dialogue devenu impossible et de reconstruire une confiance détruite.

\section{Conclusion}

Nous déduisons de ce parcours une nouvelle définition de la communication interpersonnelle réussie :

"Communiquer, c'est mettre en commun ce que nous savons d'unique, ce que nous faisons d'unique, ce que nous sommes d'unique pour découvrir nos identités uniques et notre humanité commune ».

Chaque personne constitue un tout sans équivalent au-delà des distinctions auxquelles nous avons procédé :

- celui de l'être humain en quatre composantes : l'être corporel, intellectuel, relationnel et spirituel,

- celui de la communication en quatre fonctions: transfert d'information, pilotage d'action, création de relation et découverte d'identité.

La communication contribue à faire l'unité de ces composantes de l'être, à travers ces différentes fonctions.

Un corps coupé de son intelligence, une identité déconnectée de l'action entreprise, tout cela perdrait son sens et nous jetterait dans l'absurde.

La valeur de l'être humain réside précisément dans l'intégration de ces quatre composantes corporelle, intellectuelle, relationnelle et spirituelle, qui se mettent au service les unes des autres, qui s'intègrent en une unité pleinement humaine.

Pascal, dans ses Pensées, souligne en effet que : 
«Toutes choses étant causées et causantes, aidées ou aidantes, médiates ou immédiates, et toutes s'entretiennent par un lien naturel et insensible qui lie les plus éloignées et les plus différentes, je tiens pour impossible de connaître les parties sans connaître le tout, non plus que de connaître le tout connaître particulièrement les parties ».

A l'issue de ce travail, nous percevons mieux la différence entre le « mal communiquer » et le «bien communiquer » :

- respecter ou non le caractère unique et défaillant de chaque personne

137 - négocier ou sans cesse remettre en cause la légitimité de sa place et de son action

138 - être loyal ou non dans ses engagements et ses promesses

- bien ou mal gérer la confiance en l'autre, ce qui se traduit par une prise de risque avec lui ajustée ou non. De nos choix répétés découleront le conflit ou la coopération durable, la trahison ou la réconciliation toujours possible.

\section{BIBLIOGRAPHIE}

AXELROD, Donnant-donnant. Odile Jacob, 1992. BALMARY, M., Le sacrifice interdit. Grasset 1986.

BATESON, G., Pour une écologie de l'esprit (tomes I et 11). Seuil 1977, 1980.

BIBLE de Jérusalem.

DUPUY, J-P., Common knowledge et sens commun, cahier du CREA, n 9, 1988.

FRANKO, E., De la cécité mentale et de la resilience, actes du colloque « du désastre au désir ». à paraître l'Harmattan. 2002.

GIRARD, R.. La violence et le sacré. J'ai vu Satan tomber comme l'éclair. Grasset 1972.2001.

HABERMAS, J., Théorie de l'agir communicationnel (tomes I et II). Fayard 1987.

LABORIT, H., La nouvelle grille. Gallimard 1982.

LE CARDINAL, G., Construire la confiance, une vertu nouvelle pour sortir du désir mimétique et de la violence, Communication et Organisation, $n^{\circ} 16$, juillet 2001.

LE CARDINAL, G., POUZOULLIC, B., GUYONNET, J-F.. La dynamique de la confiance. Dunod 1997, 1999, 2001.

LE CARDINAL, G., L'homme communique comme unique : modèle systémique de la communication interpersonnelle finalisé, habilitation à diriger des recherches. Université Bordeaux III, oct. 1989.

NOWAK, M., SIGMUND, K., A stategy of win-stay, lose-shift that out performs; Tit for Tat in the Prisonner's Dilemma game. Nature, vol. 364,

1993.

PASCAL, Les Pensées, les éditions G. Cres et Cie, 1928.

DE SOUZENELLE, A., L'éternel féminin. Albin Michel, 1997. 
VASSE, D., Le poids du réel, la souffrance. Seuil 1983.

WATZLAWICK, P., Une logique de communication. Seuil, 1972.

WEAVER, W., SHANNON, C, Théorie mathématique de la communication. Retz CEDL 1975

\section{NOTES}

1. Bateson. G., Pour une écologie de l'esprit. Seuil 1977.

2. de Souzenelle, A., L'éternel féminin. Albin Michel. 1997

3. Balmary. M., Le sacrifice interdit. Grasset, 1986

4. Girard, R., La violence et le sacré. Grasset, 1972

5. Axelrod. Donnant-donnant.Odile Jacob. 1992

6. Nowak. M., Sigmund. K.., Asrategy of winstay, loshift....Nature, vol 364, 1993

7. Le Cardinal, G., Guyonnet, J-F., Pouzoullic, B., La dynamique de la confiance, Dunod. 1997. 1999. 2001.

8. Description de la méthode PAT-Miroir dans : la dynamique de la confiance, Dunod, 1997, 1999, 2001

9. Le Cardinal, G., Construire la confiance..., communication et organisation, $n^{\circ} 16$, juillet 2001

10. Laborit. H., La nouvelle grille, Gallimard, 1982

11. Habermas, J.. Théorie de l'agir communicationnel, Fayard 1987

12. Dupuy, J-P., Common Knowledge..., cahiers du CREA. $n^{\circ} 9,1988$

13. Vasse, D., Le poids du réel, la souffrance. Seuil 1972

14. Franko. E., De la cécité mentale et de la resilience, colloque du désastre au désir, à paraître l'Harmattan. 2002.

15. Watzlawick. P., Une logique de la communication. Seuil 1977

16. Le Cardinal, G., L'homme communique comme unique, habilitation à diriger des recherches. Université Bordeaux III, oct.1989

17. Habermas, J.. op. cit.

18. Watzlawick. P.. op. Cit.

19. Watzlawick. P., op. Cit.

\section{RÉSUMÉS}

Une éthique de la communication, c'est-à-dire le savoir bien communiquer au service de bonnes finalités se construit sur des définitions précises de l'autonomie, de l'interaction et de la communication interpersonnelle. Mais l'étape essentielle consiste à identifier la structure unité d'interaction pour en anticiper toutes les conséquences dangereuses. On peut alors déduire de ces définitions les pièges, les principes et les précautions susceptibles de viabiliser les quatre fonctions repérées dans la pragmatique de la communication: le transfert d'information, le pilotage de l'action, la création de relation et la découverte d'identité.

The ethics of communication i.e. knowing how to communicate properly in order to reach meaningful aims, are built on precise definitions of autonomy, interaction and interpersonnal communication. However a major step consists of defining the strucure of the interaction unit in order to anticipate possible dangerous consequences of partial interpretation of relational 
breakdowns. We can therefore deduce from these definitions the pitfalls, the principles and precautions which can render secure the four functions identified in the pragmatics of human communication: the tranfer of information, the conducting of actions, the creation of relationships and the discovery of identities.

INDEX

Mots-clés : éthique, communication interpersonnelle, interaction, confiance, autonomie

\section{AUTEUR}

\section{GILLES LE CARDINAL}

Gilles Le Cardinal est professeur SIC à l'Université de Technologie de Compiègne où il dirige la formation doctorale "Sciences de l'Homme et Technologies de la Cognition et de la Coopération ». L'équipe de recherche «Communication et sécurité » qu'il anime au sein de l'équipe d'accueil COSTECH, a mis au point une méthode appelée PAT-Miroir pour construire la coopération dans les projets complexes. Elle est décrite dans un ouvrage intitulé «La dynamique de la confiance ». 